\title{
Diversity Exploration and Negative Correlation Learning on Imbalanced Data Sets
}

\author{
Shuo Wang and Ke Tang and Xin Yao
}

\begin{abstract}
Class imbalance learning is an important research area in machine learning, where instances in some classes heavily outnumber the instances in other classes. This unbalanced class distribution causes performance degradation. Some ensemble solutions have been proposed for the class imbalance problem. Diversity has been proved to be an influential aspect in ensemble learning, which describes the degree of different decisions made by classifiers. However, none of those proposed solutions explore the impact of diversity on imbalanced data sets. In addition, most of them are based on re-sampling techniques to rebalance class distribution, and over-sampling usually causes overfitting (high generalisation error). This paper investigates if diversity can relieve this problem by using negative correlation learning (NCL) model, which encourages diversity explicitly by adding a penalty term in the error function of neural networks. A variation model of NCL is also proposed - NCLCost. Our study shows that diversity has a direct impact on the measure of recall. It is also a factor that causes the reduction of F-measure. In addition, although NCL-based models with extreme settings do not produce better recall values of minority class than SMOTEBoost [1], they have slightly better performance of F-measure and G-mean than both independent ANNs and SMOTEBoost and better recall than independent ANNs.
\end{abstract}

\section{INTRODUCTION}

$\mathbf{C}$ LASS imbalance learning is the learning problem in which instances in some classes heavily outnumber the instances in other classes. Imbalanced data sets (IDS) correspond to domains that suffer this problem. Classification on IDS often gets poor results, because standard machine learning algorithms tend to be overwhelmed by the large classes and ignore the small ones. Most of those traditional classifiers operate on data drawn from the same distribution as the training data, and assume that maximizing accuracy is the principle goal [2]. However, many real-world applications encounter the problem of imbalanced data, such as medical diagnosis, fraud detection, text classification, and oil spills detection [3].

Some solutions to the class imbalance problem have been proposed at both data level [4] [5] [6] and algorithm level [7]. As one group of the solutions, ensemble systems have been drawing more and more attention because of their flexible characteristics for imbalanced data. For example, some existing popular models include BEV (Bagging Ensemble Variation) [8], SMOTEBoost [1], and DataBoost [9].

Shuo Wang and Xin Yao are with the School of Computer Science, The University of Birmingham, Birmingham, UK (email: \{s.wang, x.yao\}@cs.bham.ac.uk). Ke Tang is with the Nature Inspired Computation and Applications Laboratory, the Department of Computer Science and Technology, University of Science and Technology of China, Hefei, Anhui, China. (email: ketang@ustc.edu.cn).
They are proposed based on Bagging [10] or Boosting [11] by improving their re-sampling methods. Re-sampling allows to re-balance class distribution, in order to improve classification accuracy of each classifier on minority class. However, overfitting is easily caused by over-sampling on minority class instances in training data, because of too much duplication from very few instances, especially for nonlinear classification techniques, such as decision tree and neural networks. Although the proposed models try to avoid this problem by using ensemble, it is still unclear why they can make such improvement. In addition, the data generation techniques used in the above ensemble models may suffer performance degradation as training data has high complexity, such as complex data distribution and multiple minority classes. We are aiming at an alternative better solution from algorithm level.

Ensemble of multiple classifiers is expected to reduce generalisation error by considering the opinions from multiple classifiers. Therefore, diversity becomes an important factor. If every classifier gives the same opinion, constructing multiple classifiers becomes meaningless. Overfitting may reduce the power of ensemble. Diversity is the degree to which classifiers make different decisions on one problem. Diversity allows voted accuracy to be greater than that of single classifier. Among above ensemble solutions for imbalanced data sets, we suggest that it might be diversity that leads to performance improvement more or less. Therefore, we are interested in the issue that how diversity affects classification performance especially on minority classes. Understanding diversity on minority class helps us improve ensemble solutions. Experiments in the following sections show that increasing diversity potentially allows more instances belonging to minority class to be found, and the best overall performance requires proper diversity degree.

Negative Correlation Learning (NCL) [12] is an ensemble learning method of neural networks that uses different mechanism to induce diversity. It has shown a number of empirical successes on various applications and competitive results with other methods like Bagging and Boosting [10] [11]. NCL encourages diversity directly by changing its error function into "diversity-encouraging" error function [13]. To further explore the potential of diversity on the class imbalance problem and avoid sensitivity to data distribution, we compare four neural network ensemble models including two NCL-based solutions, an independently constructed ANNs, and a popular over-sampling based model SMOTEBoost [1]. We expect NCL can improve performance especially on the classification of minority class. A NCL 
variation, NCLCost, is proposed motivated by the different costs between minority class and majority class. No related research has been working on this problem. Most of the current studies choose decision tree as learning algorithm in the area of class imbalance learning [14]. Although there are some papers comparing several learning methods, they only apply to single classifier [3].

In this paper, therefore, the goal is to discover the power of ensemble diversity on imbalanced data sets, especially by using neural networks as base learner. Two main research issues are explored:

- How does diversity impact ensemble performance on imbalanced data sets, especially the classification of minority class?

- Is NCL able to improve performance of minority classification? Does NCL have better generalisation ability on imbalanced data sets compared to independently trained ANNs and SMOTEBoost?

To the first issue, we observe performance tendency under different diversity degree by controlling sampling rate from under-sampling to over-sampling on six two-class data sets. Bagging ensemble technique is used. Therefore, "UnderBagging" and "OverBagging" are constructed. More detailed study of this issue can be found in our previous work [15]. To the second issue as the further work of the first issue, four neural network ensemble models are constructed and compared - independent neural networks (ANNs), standard NCL model (NCL), NCLCost, and SMOTEBoost. It is still a novel topic that is worth exploring in the class imbalance area. It is the main contribution of this paper. When looking into these two issues, four evaluation measures are selected: recall, F-measure, G-mean [16], and Q-statistics [17].

This paper is further organized as follows: section II presents related work, section III describes the proposed experimental design including two NCL-based ensemble models, section IV gives observations from experiments and analysis of experimental results, and section $\mathrm{V}$ presents the conclusions of the work.

\section{RELATED WORK}

To deal with class imbalance problem, several improved ensemble algorithms have been proposed that outperform single classifiers and standard ensemble methods. Firstly, subsection A introduces some successful ensemble learning techniques for imbalanced data set existing in the literature. Then, subsection B describes the theoretical background and the idea of negative correlation learning used in this work.

\section{A. Imbalanced Ensemble Learning}

Existing imbalanced ensemble solutions emphasize the choice of re-sampling techniques. Over-sampling or undersampling is applied before constructing ensemble classifiers, in order to balance class distribution. Among a number of re-sampling techniques, random over-sampling and random under-sampling are the simplest ones to be applied by duplicating or eliminating instances randomly. To avoid overfitting of random oversampling, SMOTE is proposed by Chawla [18], which is a popular method of over-sampling by generating synthetic instances. However, it may be sensitive to the complexity of training data.

Based on those re-sampling methods, there are some ensemble methods, such as SMOTEBoost [1], Data-Boost [9], and BEV [8]. The first two improve Boosting by combining data generating methods. Instead of changing the distribution of training data by updating the weights associated with each instance in standard Boosting, SMOTEBoost alters the distribution by adding new minority-class instances that applies the SMOTE algorithm. Experimental results indicate that this approach allows SMOTEBoost to achieve higher Fvalues than standard Boosting and SMOTE algorithm with a single classifier. DataBoost aims at improving performance of minority class and majority class at the same time. Hard instances from both majority class and minority class are identified. BEV uses Bagging by under-sampling majority class instances. However, BEV was only tested on one realworld application and there is not comparison with other models. SMOTEBoost and DataBoost have been proved experimentally to have better performance than standard Boosting algorithm, but they need further experiments on more complex data sets since new training instances are generated.

Considering the above discussion, although a number of researchers have been working on this topic, very few discuss the diversity or give us a clear idea about "why do the authors believe that ensemble model can improve performance of minority?" Since diversity and accuracy are the two fundamental aspects of ensemble. Diversity analysis on single class is an interesting issue. To further explore the generalisation ability of diversity, NCL is taken into consideration from algorithm level.

\section{B. Theoretical Background of NCL}

The idea of negative correlation learning (NCL) is to manage diversity explicitly by adding emphasis on the covariance between the individual classifiers to the error function and training members together. Its theoretical evidence is the bias-variance-covariance tradeoff and ambiguity decomposition [12] [13] shown in the equation (1) and (2).

$$
\begin{gathered}
E\left\{(\bar{f}-t)^{2}\right\}=\overline{\text { bias }}^{2}+\frac{1}{M} \overline{v a r}+\left(1-\frac{1}{M}\right) \overline{\text { covar }} \\
(\bar{f}-t)^{2}=\frac{1}{M} \sum_{i}\left(f_{i}-t\right)^{2}-\frac{1}{M} \sum_{i}\left(f_{i}-\bar{f}\right)^{2}
\end{gathered}
$$

where $\bar{f}$ is the combination (averaged result) of the $M$ component classifiers; $\overline{\text { bias }}$ is the averaged bias of the classifiers; $\overline{v a r}$ is the averaged variance; $\overline{c o v a r}$ is the averaged covariance.

Equation (1) is the decomposition of the generalisation error of an ensemble and it tells us it is dependent on the covariance between the individuals. The covariance, the third term in the equation (1), is expressed as: 
$\overline{c o v a r}=\frac{1}{M(M-1)} \sum_{i} \sum_{j \neq i} E\left\{\left(f_{i}-E\left\{f_{i}\right\}\right)\left(f_{j}-E\left\{f_{j}\right\}\right)\right\}$

Equation (2) is the quadratic error of ensemble at a single arbitrary data point with the assumption of uniform weighting. The second term is referred to as the Ambiguity.

The first two equations express the effect of correlations on ensemble error. It illustrates that the generalisation error of an ensemble also depends on covariance between the individuals in addition to the bias and variance of each individual classifier. Moreover, diversity cannot be maximized simply without affecting the other parts of error. In fact, the more diverse the members are, the more well spread will their outputs be around the target value resulting in the expected value of the member outputs being closer to this target value [19]. For imbalanced data, this could be a potential advantage. Minority class data is always learned too much. It is expected that diversity can bring more chance bias to minority class classification.

However, NCL has not been explored in class imbalance learning area. Several questions are raised: when data from one class is very scarce, does NCL still work better than other ensembles? Is it possible that NCL can improve classification on minority class? The methods used and proposed in this paper are trying to explore this topic by manipulating NCL algorithm on minority class instances.

\section{EXPERIMENTAL DESIGN}

This section presents our experimental design for diversity and NCL analysis. Firstly, we implemented Bagging ensemble algorithm combined with randomly re-sampling methods, referred to as UnderBagging and OverBagging. Sampling strategy used in the experiments is described. The aim is to observe the relationship between diversity and classification performance. The reason that we select Bagging as our ensemble method is for the convenience of observing performance tendency by manipulating one variable - sampling rate. Weight updating rule in Boosting may bring confusion. Secondly, algorithms of two NCLbased ensemble models are presented. NCLCost is the "costsensitive" version of NCL.

Suppose there are $C$ classes. The $i$-th class has $N_{i}$ number of training instances. Those classes are sorted by $N_{i}$ such that for the $i$-th class and the $j$-th class, if $i<j$ then $N_{i} \leq$ $N_{j}$. Therefore, $N_{C}$ is the maximum number of instances belonging to one class. $M$ classifier members are built, $k=$ $1,2, \ldots, M$.

\section{A. Bagging Variations: from UnderBagging to OverBagging}

We now construct each classifier in ensemble iteratively using subset $S_{k}$ from training set $S$. In UnderBagging, each subset $S_{k}$ is created by under-sampling majority classes randomly with replacement to construct the $k$-th classifiers. In the similar way, OverBagging forms each subset simply by over-sampling minority classes randomly. After construction, majority vote is performed when a new instance comes. Each classifier gives its judgment. Final classification decision follows the most voted class. If a tie appears, then the class with minor instances is returned. The whole procedure could be described into 3 steps -"sampling, constructing ensemble, voting" from training phase to testing phase. The basic idea is that we maintain a variable - sampling rate $a \%$, in order to change the size of training subset and keep minority instances and majority instances balanced. Assume class $i$ is treated as minority with number $N_{i}$, and other classes are merged as majority with number $N_{m a j}=N_{1}+N_{2}+\ldots+N_{C}-N_{i}$. If the current subset $S_{k}$ has $N_{m a j} \cdot a \%$ majority instances, then the size of minority class is $N_{i} \cdot\left(N_{m a j} / N_{i}\right) \cdot a \%$. When $a$ is small, the ensemble model is UnderBagging. Otherwise, it is OverBagging. The strategy detail is shown in Table I. The work in this paper only considers two-class cases.

TABLE I

From UNDERBAGGING TO OVERBAGGING

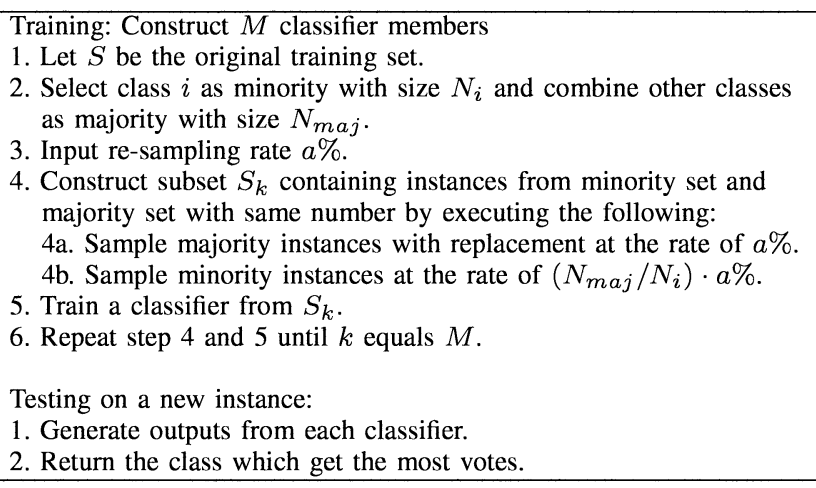

In this experiment, sampling rate ' $a$ ' is set at multiple values of 10 . In this way 10 ensembles are obtained from one data set. Smaller ' $a$ ' may lead to data subsets that are less overlapped with one another and thus result in more diverse ensemble system. The relationship between sampling and diversity is explained in Section IV.

\section{B. NCL-based Models}

Given the fact that similar members preclude the need for ensembles, classifiers have to be diverse. NCL is known for its capability of encouraging diversity explicitly while maximizing accuracy of individual classifiers. In addition, a problem exists in class imbalance field, which is oversampling causes overfitting on minority instances. Considering that NCL tends to spread outputs more around the target value and aims at better generalisation ability, we expect that it could relieve the overfitting problem. Four ANN ensemble models are constructed: ensemble with independent members (bootstraping), SMOTEBoost with ANN base learners, ensemble by using standard NCL algorithm, and an NCL variation referred to as "NCLCost". NCLCost is motivated by different costs among the classes in imbalanced data sets. Minority class needs less specific classification boundary, while majority class needs more accurate boundary. 
1) NCL: Negative correlation learning [12] is a neural network ensemble learning technique developed in the Evolutionary Computation literature. Negative correlation learning introduces a correlation penalty term into the error function of each individual network in the ensemble so that all the networks can be trained simultaneously and interactively on the same training data set $S$. The error function $e_{i}$ for network $i$ in negative correlation learning is defined by:

$$
e_{i}=\frac{1}{2}\left(f_{i}-t\right)^{2}+\lambda\left(f_{i}-\bar{f}\right) \sum_{j \neq i}\left(f_{j}-\bar{f}\right)
$$

The first term in the right side is the empirical risk function of network $i$. The second term is a correlation penalty function. The purpose of minimizing the penalty term is to negatively correlate each network's error with errors from the rest of the ensemble. During the training process, each network $i$ not only minimizes the difference between its output and expected output, but also maximizes the difference between ensemble output and individual output, so that diversity and accuracy are guaranteed at the same time.

2) NCLCost: NCLCost has the same idea as NCL, but different objectives. NCL applies penalty term on every instance in the training set to achieve better performance in "full scale". Because of the properties of imbalance distribution, classifiers exerting different effect on each class are needed. Otherwise, it may still tend to classify an instance as majority and overfit minority class. Considering this point, NCLCost treats minority data and majority data in different ways, which makes NCL ensemble system "cost-sensitive" by managing the penalty strength $\lambda$ in penalty term. For example in a two-class data set, higher $\lambda$ value, such as 1 , is assigned to minority data; small $\lambda$ value, such as 0 , is assigned to majority data. Therefore, the goal is to guarantee both accuracy and diversity of minority class and maximize only accuracy of majority class. For a data set with multiple classes, different $\lambda$ values can be chosen depending on class costs. The algorithm is presented in Table II. A random over-sampling is performed firstly to balance data distribution in step 2 before NCL training phase starts. In fact, all the ANN-based models in our experiments, apart from SMOTEBoost model which has new data generated, are built after training subsets are re-balanced. It should not influence the results, since they use the same sampling rate. Some preliminary experiments are also implemented. NCL model without sampling gives very poor results on highly imbalanced data sets.

In our current experiments, the extreme case is tested, in which ' $a$ ' is equal to 1 and ' $b$ ' is equal to 0 .

\section{EXPERIMENTAL ANALYSIS}

This section presents the experiments involving diversity analysis and NCL exploration. Therefore, experiments include two main parts. Firstly, observations of performance tendency are given by using the Bagging variations described in Table I. Secondly, four ANNs ensemble models are
TABLE II

\section{PSEUDOCODE FOR NCLCOST}

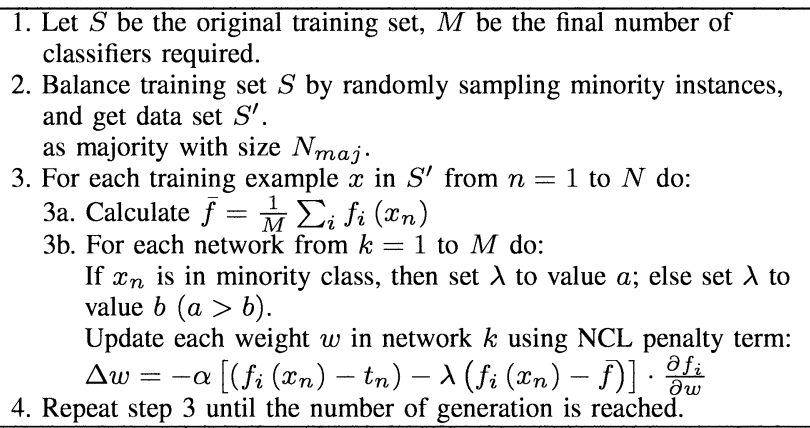

discussed, including the comparisons between two NCLbased models and the others.

\section{A. Data Sets and Experimental Setup}

Our experiments use eight UCI data sets including six two-class and two multi-class - "Yeast" with 10 classes and "Abalone" with 28 classes, which are shown in Table III. The six two-class data sets are used in diversity analysis experiments. Imbalance rate varies from to $45 \%$ (slightly imbalanced) to $34 \%$ (imbalanced) (percentage of minority class data in whole data set). Performance curves are depicted according to outputs from each diversity degree. The two multi-class data sets are converted into five two-class problems, which means a target class from data set is chosen as minority and others are merged as majority. These generated sets are highly skewed with imbalance rate from $6 \%$ to $17 \%$. All the five data sets are used in the comparison among four neural network ensemble models.

TABLE III

Experimental Data Sets (Imbalance Rate is the PERCEntage of MINORITY CLASS INSTANCES IN THE WHOLE DATA SET)

\begin{tabular}{|c|c|c|c|c|}
\hline Data Set & Size & Attributes & Minority Class & Imbalance Rate \\
\hline Hepatitis & 155 & 19 & "2" & $45 \%$ \\
\hline Heart & 270 & 13 & "2" & $44 \%$ \\
\hline Liver & 345 & 6 & "1" & $42 \%$ \\
\hline Pima & 768 & 8 & "1" & $35 \%$ \\
\hline Ionosphere & 351 & 34 & "b" & $35 \%$ \\
\hline Breast-w & 699 & 9 & "4" & $34 \%$ \\
\hline Yeast & 1484 & 8 & "ME3" & $11 \%$ \\
\cline { 4 - 5 } & & & "MIT" & $16.4 \%$ \\
\hline Abalone & \multirow{2}{*}{4177} & \multirow{2}{*}{8} & "6" & $6.2 \%$ \\
\cline { 3 - 5 } & & & "7" & $9.4 \%$ \\
\cline { 3 - 5 } & & & "8" & $13.6 \%$ \\
\hline
\end{tabular}

The parameters used in the experiments are chosen after visual inspection of some preliminary executions. In diversity analysis in subsection B for the efficiency consideration, C4.5 decision tree is chosen as base learner. 10-fold cross validation is performed on each data set by running 30 times. The results are the average of 30 runs of 10 folds. Each ensemble model creates 21 classifier members. In subsection $\mathrm{C}, 10$ runs of 10-fold cross validation are performed with learning rate 0.3 in neural networks' training. Every ensemble model 
consists of 8 ANNs combined with majority voting. The results are confirmed by $\mathrm{T}$ test with $95 \%$ of confidence. For now we choose two boundary values 0 and 1 as the penalty strength $\lambda$, in order to observe the extreme situations caused by diversity. However, further detailed study with different learning rate and penalty strength is necessary and important as our future work. In SMOTEBoost model construction, parameters $k$ and $N$, which denote $k$-Nearest neighbor and the amount of generated instances, need to be set manually. $k$ is set to 3 and $N$ is set to 100,200 and 300 respectively in the implementation. The results of SMOTEBoost shown in the following comparison table are those with the highest F-measure outputs.

Class imbalance has its own evaluation criteria for single class and overall performance. When performance of single class is evaluated, recall, precision, and F-measure are commonly used. Recall tells us how many minority class instances are identified, but may sacrifice system precision by misclassifying majority class instances. Value of F-measure (or F-value) incorporates both precision and recall, in order to measure the "goodness" of a learning algorithm for the class. For evaluating overall performance, geometric mean (G-mean) and ROC analysis are better choices [16]. In this work, we choose recall, precision, F-measure and G-mean value to depict performance tendency curves at different diversity degrees and compare ensemble models. Q-statistics is selected as our diversity measure because of its easily understood form [17]. For two classifiers $L_{i}$ and $L_{k}$, Qstatistic value is,

$$
Q_{i, k}=\frac{N^{11} N^{00}-N^{01} N^{10}}{N^{11} N^{00}+N^{01} N^{10}}
$$

where $N^{a b}$ is the number of training instances for which $L_{i}$ gives result ' $a$ ' and $L_{k}$ gives result ' $b$ ' (It is supposed that the result here is equal to 1 if an instance is classified correctly and 0 if it is misclassified). Then for an ensemble system with a group of classifiers, the averaged Q-statistics is calculated to express the diversity over all pairs of classifiers,

$$
Q_{a v}=\frac{2}{M(M-1)} \sum_{i=1}^{M-1} \sum_{k=i+1}^{M} Q_{i, k}
$$

For statistically independent classifiers, the expectation of $\mathrm{Q}$-value is 0 . $\mathrm{Q}$-value varies between -1 and 1 . It will be positive if classifiers tend to recognize the same instances correctly, and will perform negative if they commit errors on different instances [20]. The larger the value is, the less diverse the classifier members are.

\section{B. Diversity Analysis and Performance Tendency}

1) Relationship between Diversity and Sampling: Before our experiments, we need to clarify the relationship between sampling and diversity. Our diversity analysis is based on the adjustment of sampling rate in ensemble models. However, they are not the same concept. When sampling rate changes, the size of each subset for training individual classifier gets larger or smaller. Accuracy of each classifier and diversity are varying at the same time, because more instances are injected in training phase. Therefore, when we analyze the diversity in the next section, we did not ignore the influence of accuracy. To discriminate accuracy and diversity, we use the algorithm shown in Table I on single classifier firstly, and adjust sampling rate in the same way. The result shows the relationship between sampling and accuracy before we do the diversity analysis. Figure 1 helps us to observe the difference between single classifier and ensemble with multiple classifiers. The left graph illustrates increasing tendency of output values (Recall and F-measure of minority, G-mean) by using single classifier in data set Breast-w. If we only build one classifier, classifier accuracy increases without diversity involved, caused by sampling. It results in the improvement of other measures. Other data sets have similar results, which fluctuate in a much lower range than those in ensemble.

2) From UnderBagging to OverBagging: By running the algorithm in Table $I$ on the first six two-class data sets shown in Table III, we get the tendency curves to show the changes of each measure. For the space consideration, only outputs from data set Breast-w are shown in Figure 1 with comparison between single classifier and classifier ensemble. Other data sets produce similar tendencies, except data set Ionosphere. $\mathrm{X}$-axis presents the sampling percentage from 10 to 100 , and $Y$-axis presents the average values of final outputs. Q-statistic values of minority class and whole data set are both increasing as sampling parameter ' $a$ ' becomes larger and larger, which means diversity is decreasing. More information is discussed in paper [15].

From the right graph in Figure 1, it is evident that the recall value of minority class keeps decreasing when diversity becomes smaller and smaller. The recall value of majority class performs in the opposite way, which keeps increasing. Diversity has a direct and explicit impact on recall value. Recall value, however, can only tell us how many minority instances could be found (hit rate). F-measure and G-mean are more meaningful for most real world problems. As we can observe, they both have significant improvement at the first few points, and the best value appears at neither the last point with lowest diversity degree nor the first point with highest diversity degree.

The behavior of recall value is easy to understand. Higher diversity gives more chance to find out minority instances, and vice versa. Compared with single classifier in the left graph, diversity exerts more significant influence on minority class than majority class. An instance is more likely to be classified as minority when accuracy is low. Therefore, recall of minority is comparatively high. As accuracy on majority and minority becomes higher, diversity goes down. High accuracy on minority causes overfitting, which means low diversity and low recall. Too much duplication lowers the generalisation ability of minority class. The reason that why the best F-measure and G-mean values are not the case with highest sampling rate is because the decreasing of diversity becomes faster than the increasing of accuracy in the latter period of the curve. From the graph, precision of minority 

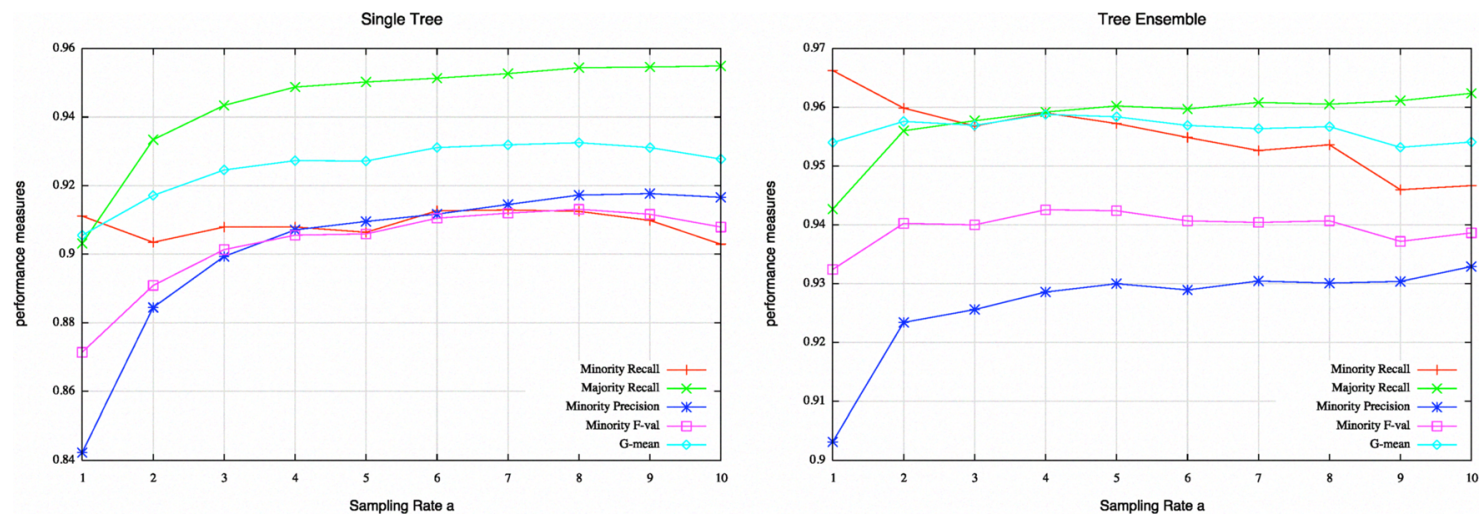

Fig. 1. Performance tendency of data set "Breast-w" by using single classifier and decision tree ensemble. X-axis: the sampling rate from 10 to 100 ; Y-axis: Y-axis: the averaged values of final outputs from 30 runs of 10 -fold cross validation.

TABLE IV

Performance Averages and Standard Deviations of 10 runs of 10-fold Cross-Validation of Independent ANNs, NCL and NCLCost Ensemble Models. The row Shows the aVerage measures of EaCh Data SET.

\begin{tabular}{|c|c|c|c|c|c|}
\hline \multicolumn{5}{|c|}{ Recall of Minoriy } \\
\hline Model/Target & ME3 & MIT & 6 & 7 & 8 \\
\hline ANNs & $0.88250 \pm 0.00823$ & $0.73958 \pm 0.01760$ & $0.88240 \pm 0.00947$ & $0.86179 \pm 0.00778$ & $0.75768 \pm 0.01731$ \\
\hline NCL & $0.90688 \pm 0.01299$ & $0.68625 \pm 0.02764$ & $0.90920 \pm 0.01237$ & $0.90590 \pm 0.00880$ & $0.76804 \pm 0.03633$ \\
\hline NCLCost & $0.91188 \pm 0.01365$ & $0.73750 \pm 0.02538$ & $0.89280 \pm 0.00860$ & $0.87179 \pm 0.01470$ & $0.75321 \pm 0.01947$ \\
\hline \multicolumn{7}{|c|}{ F-measure of Minoriy } \\
\hline Model/Target & ME3 & MIT & 6 & 7 & 8 \\
\hline ANNs & $0.73386 \pm 0.01264$ & $0.55786 \pm 0.01516$ & $0.37420 \pm 0.00319$ & $0.39744 \pm 0.00444$ & $0.37855 \pm 0.00642$ \\
\hline NCL & $0.71104 \pm 0.01402$ & $0.59205 \pm 0.01493$ & $0.35478 \pm 0.00696$ & $0.36062 \pm 0.00537$ & $0.35968 \pm 0.01374$ \\
\hline NCLCost & $0.72658 \pm 0.01123$ & $0.56760 \pm 0.01384$ & $0.36144 \pm 0.00789$ & $0.39435 \pm 0.00401$ & $0.37385 \pm 0.00528$ \\
\hline \multicolumn{7}{|c|}{ G-mean } \\
\hline Model/Target & ME3 & MIT & 6 & 7 \\
\hline ANNs & $0.90760 \pm 0.00469$ & $0.77807 \pm 0.01006$ & $0.84868 \pm 0.00438$ & $0.80023 \pm 0.00427$ & $0.70051 \pm 0.00740$ \\
\hline NCL & $0.91217 \pm 0.00649$ & $0.77112 \pm 0.01138$ & $0.84796 \pm 0.00407$ & $0.78145 \pm 0.00314$ & $0.67071 \pm 0.01257$ \\
\hline NCLCost & $0.91789 \pm 0.00727$ & $0.78120 \pm 0.01204$ & $0.84642 \pm 0.00406$ & $0.79938 \pm 0.00410$ & $0.69014 \pm 0.00844$ \\
\hline
\end{tabular}

TABLE V

Diversity Averages and Standard Deviations of 10 Runs of 10-Fold Cross-Validation of IndePEndent ANNs, NCL AND NCLCost Ensemble Models. The Row Shows the aVerage MEASURES of EACH DATA SET.

\begin{tabular}{|c|c|c|c|c|c|}
\hline \multicolumn{5}{|c|}{ Q-statistics of Minoriy } \\
\hline Model/Target & ME3 & MIT & 6 & 7 & 8 \\
\hline ANNs & $0.89051 \pm 0.04723$ & $0.95030 \pm 0.02421$ & $0.93347 \pm 0.05737$ & $0.96694 \pm 0.01270$ & $0.94969 \pm 0.00629$ \\
\hline NCL & $0.85768 \pm 0.05104$ & $0.55006 \pm 0.02976$ & $0.37382 \pm 0.06023$ & $0.07267 \pm 0.04995$ & $0.00041 \pm 0.08053$ \\
\hline NCLCost & $0.93151 \pm 0.03763$ & $0.96714 \pm 0.01395$ & $0.73585 \pm 0.09293$ & $0.79387 \pm 0.05604$ & $0.96170 \pm 0.01056$ \\
\hline \multicolumn{7}{|c|}{ Overall Q-statistics } \\
\hline Model/Target & ME3 & MIT & 6 & 7 & 8 \\
\hline ANNs & $0.98129 \pm 0.00419$ & $0.94422 \pm 0.00469$ & $0.99354 \pm 0.00064$ & $0.98716 \pm 0.00162$ & $0.94710 \pm 0.00366$ \\
\hline NCL & $0.91647 \pm 0.02863$ & $0.14557 \pm 0.03893$ & $0.44762 \pm 0.07032$ & $0.08377 \pm 0.04584$ & $-0.09503 \pm 0.03348$ \\
\hline NCLCost & $0.99162 \pm 0.00627$ & $0.93872 \pm 0.01704$ & $0.83544 \pm 0.05468$ & $0.82106 \pm 0.08088$ & $0.95421 \pm 0.01057$ \\
\hline
\end{tabular}


does not reduce along with the decrease of sampling. Its increasing tendency slows down after the third point. Diversity becomes a leading factor which causes the degradation of F-measure and G-mean. The trade-off between accuracy of each classifier and ensemble diversity decides the final performance of an ensemble [21].

\section{Independent ANNs Vs. NCL-based Models}

This section presents the comparison among independent ANNs, NCL and NCLCost models, in order to observe the impact of diversity brought by NCL. It shows that NCL obtains higher recall values but sacrifices too much accuracy because of diversity, which leads to poor F-measure and G-mean values. NCLCost shows competitive results with independent model on F-measure and G-mean, and slightly better recall values. Table IV shows the averages and standard deviations of three evaluation metrics (recall of minority, F-measure of minority, and G-mean) on testing data (generalisation). They are derived from 10 runs of $10-$ fold cross-validation, and $\mathrm{T}$ test with $95 \%$ of confidence is applied. Significance analysis is presented in Table VII.

1) Independent ANNS Vs. NCL: In the five very skewed cases, three out of five have significant improvement of recall value on minority class, which means more minority data is identified. However, four out of five cases in NCL model have significant reduction of F-measure value due to the low accuracy. This also leads to unsatisfactory results on G-mean, where there is only two cases ANNs wins and three ties. A significant reduction of Q-statistic values is observed in Table $\mathrm{V}$. Nine out of ten cases including both minority and overall in NCL model are more diverse than independent ANNs on testing data.

The experiments presented in this section indicate that NCL does bring diversity into ensemble and achieve higher recall values. When the model gets less overfitting, accuracy on majority class is sacrificed. This problem is unavoidable due to the trade-off between diversity and accuracy. This extreme case when penalty strength is set to 1 is not the optimum point we expect and not suitable to class imbalance learning in some cases. Therefore, the selection of a more suitable parameter will be covered as our future work. We expect optimal parameter allows the use of NCL model more suitable to class imbalance learning.

2) Independent ANNs Vs. NCLCost: NCLCost is a "softer" way than NCL. The idea of NCLCost is to treat minority data and majority data in different ways, so as to make the learning model more diverse on minority data and more accurate on majority data. From performance comparison in Table IV, NCLCost shows some advantages. It gets better recall values in some cases and never loses. Independent ANNs and NCLCost have competitive results on F-measure and G-mean, in which most of the cases are ties. However, we cannot see too much difference on Qstatistics in Table V. NCLCost does not bring diversity as much as we expect. Only two cases, class "6" in Abalone selected as minority class and class "7" in Abalone selected as minority class, get more diverse results in NCLCost model. The other three produce similar or even less diverse values in NCLCost than those in independent ANNs. The explanation that can be found is that NCLCost is better generalised than ANNs during training phase. Less specific classification boundary towards minority class is achieved. It is also possible that complexity of training data influences Q-statistic values. Classifiers are more likely to make same judgement on testing data. Further investigation is necessary. For example, different diversity measures could be chosen, in order to check if the same thing happens.

In summary, the two comparisons in subsection $\mathrm{C}$ show that diversity has direct influence on recall, which is consistent with the results shown in subsection B "Diversity Analysis". Secondly, NCL sacrifices accuracy too much resulting in poor overall performance, while NCLCost achieves similar overall performance to independent ANNs and slightly better recalls on minority class. NCLCost tends to balance accuracy and recall. Currently, the two NCL-based models in the experiments have extreme parameter settings on penalty strength. Therefore, thirdly, it is worth to explore optimal parameter aiming at a better NCL-based model in class imbalance learning as a future topic.

TABLE VI

Performance Averages and Standard Deviations of 10 Runs of 10-Fold CRoss-VAlidation of SMOTEBoost. THE COLUMN SHOWS THE AVERAGE MEASURES OF EACH DATA SET.

\begin{tabular}{|c|c|c|c|}
\hline Target & Recall of Minority & F-measure of Minority & G-mean \\
\hline ME3 & $0.95375 \pm 0.01619$ & $0.74223 \pm 0.01500$ & $0.93702 \pm 0.00977$ \\
\hline MIT & $0.86667 \pm 0.03037$ & $0.50182 \pm 0.01098$ & $0.74990 \pm 0.00966$ \\
\hline 6 & $0.92840 \pm 0.02533$ & $0.35134 \pm 0.01468$ & $0.84578 \pm 0.02033$ \\
\hline 7 & $0.87333 \pm 0.03604$ & $0.38447 \pm 0.01718$ & $0.78055 \pm 0.03134$ \\
\hline 8 & $0.82482 \pm 0.01121$ & $0.34869 \pm 0.01040$ & $0.64364 \pm 0.01472$ \\
\hline
\end{tabular}

TABLE VII

T-TEST COMPARISON OF IMPORTANT MEASURES WITH CONFIDENCE LEVEL AT 95\%. EACH TABULAR SHOWS THE AMOUNT OF WIN-TIE-LOSE OF NCL AND NCLCOST IN A COLUMN COMPARING WITH INDEPENDENT ANNS AND SMOTEBOOST IN A ROW.

\begin{tabular}{|c|c|c|c|c|c|c|}
\hline & \multicolumn{2}{|c|}{ Recall of Minoriy } & \multicolumn{2}{c|}{ F-measure of Minority } & \multicolumn{2}{c|}{ G-mean } \\
\cline { 2 - 7 } & NCL & NCLCost & NCL & NCLCost & NCL & NCLCost \\
\hline ANNs & $3-1-1$ & $2-3-0$ & $1-0-4$ & $0-4-1$ & $0-3-2$ & $1-3-1$ \\
\hline SMOTEBoost & $1-0-4$ & $0-1-4$ & $1-2-2$ & $2-2-1$ & $2-2-1$ & $2-2-1$ \\
\hline
\end{tabular}

\section{SMOTEBoost Vs. NCL-based Models}

Both SMOTEBoost and NCL-based models aim at better performance on IDS. The difference between them is that SMOTEBoost introduces diversity and broadens classification boundary towards the majority side by generating new instances, while NCL-based models try to bring diversity from algorithm level by using original data. The two different strategies are compared in this section. Table VI presents 
the measure outputs of the SMOTEBoost ensemble. One issue that needs to be mentioned is that the performance of SMOTEBoost is sensitive to the chosen parameters ' $N$ ' (the amount of generated examples) and ' $k$ ' (k-nearest neighbor). Thus, $N$ is set to 100,200 and 300 in this work, and $k$ is set to 3. The result with the highest F-measure values is chosen in the comparison. The following analysis gives more attention to F-values and G-mean.

SMOTEBoost gets much better recall values than NCLbased models in most cases. NCLCost shows slightly better F-measure and G-mean, and NCL has competitive results. In Table VII, both NCL and NCLCost have two significantly better G-mean values out of five cases, while SMOTEBoost wins once. Considering F-measure, NCLCost performs slightly better and NCL performs slightly worse than SMOTEBoost. From this point, SMOTEBoost is a potentially better choice. However, controlling the balance among recall, F-measure and G-mean of SMOTEBoost becomes a crucial step. The key point is to decide the amount of new instances to be generated. In addition, it tends to be sensitive to the complexity of training data. NCL-based model does not have these problems, because they bring diversity from the algorithm level. The only parameter is the coefficient in penalty term to present the relative importance of each class. It is also easy to expand NCL-based models to multi-class cases.

\section{Conclusions}

The paper explores two main research issues. The impact of diversity in class imbalance learning is firstly studied empirically on six UCI data sets by using the Bagging variation models. The results suggest that diversity influences recall value significantly and degrades F-measure and Gmean in some situations. Generally, larger diversity causes better recall for minority, but worse recall for majority classes. For other measures, the best F-measure value and G-mean value don't appear at the status with high accuracy / low diversity or the status with low accuracy / high diversity shown in the curves. Proper diversity degree results in better performance. Then, considering the results from the diversity analysis, we propose to use NCL to improve diversity to some extent. We compare four neural network ensemble models, two of which are NCL-based. Compared to independent ANNs, it shows that NCL obtains higher recall values, but sacrifices too much accuracy because of diversity. NCL model with extreme parameter setting is not suitable to class imbalance classification. NCLCost is a "softer" way that produces competitive results with independent ANNs on F-measure and G-mean, and better recall values. Compared to SMOTEBoost, NCL and NCLCost get similar or slightly better performance of F-measure and G-mean, but worse recall of minority. It is worth to explore more about how to achieve a better NCL-based model with optimal parameter in class imbalance learning as future work.

\section{ACKNOWLEDGMENT}

This work was partially supported by an Overseas Research Student Award (ORSAS) and a scholarship from the School of Computer Science, University of Birmingham, UK, and a National Natural Science Foundation of China Grant (No. 60802036).

\section{REFERENCES}

[1] N. V. Chawla, A. Lazarevic, L. O. Hall, and K. W. Bowyer, "Smoteboost: Improving prediction of the minority class in boosting," in Knowledge Discovery in Databases: PKDD 2003, vol. 2838/2003, 2003, pp. 107-119.

[2] N. V. Chawla, N. Japkowicz, and A. Kotcz, "Editorial: Special issue on learning from imbalanced data sets," SIGKDD Explor. Newsl., vol. 6, no. 1, pp. 1-6, 2004.

[3] N. Japkowicz and S. Stephen, "The class imbalance problem: A systematic study," Intelligent Data Analysis, vol. 6, no. 5, pp. 429 - 449, 2002.

[4] G. E. A. P. A. Batista, R. C. Prati, and M. C. Monard, "A study of the behavior of several methods for balancing machine learning training data," Special issue on learning from imbalanced datasets, Sigkdd Explorations, vol. 6, no. 1, pp. 20-29, 2004.

[5] R. Barandela, R. M. Valdovinos, J. S. Sanchez, and F. J. Ferri, "The imbalanced training sample problem: Under or over sampling?" Lecture Notes in Computer Science, vol. 3138, pp. 806-814, 2004.

[6] Z.-H. Zhou and X.-Y. Liu, "Training cost-sensitive neural networks with methods addressing the class imbalance problem," in IEEE Transactions on Knowledge and Data Engineering, vol. 18, no. 1, 2006, pp. 63- 77.

[7] N. Japkowicz, C. Myers, and M. A. Gluck, "A novelty detection approach to classification," in IJCAI, 1995, pp. 518-523.

[8] C. Li, "Classifying imbalanced data using a bagging ensemble variation," in ACM-SE 45: Proceedings of the 45th annual southeast regional conference, 2007, pp. 203-208.

[9] H. Guo and H. L. Viktor, "Learning from imbalanced data sets with boosting and data generation: the databoost-im approach," SIGKDD Explor. Newsl., vol. 6, no. 1, pp. 30-39, 2004.

[10] L. Breiman, "Bagging predictors," Machine Learning, vol. 24, no. 2, pp. 123-140, 1996.

[11] Y. Freund and R. E. Schapire, "Experiments with a new boosting algorithm," in Proc. of the 13th. Int. Conf. on Machine Learning, 1996, pp. $148-156$.

[12] Y. Liu and X. Yao, "Ensemble learning via negative correlation," Neural Networks, vol. 12, no. 10, pp. 1399-1404, 1999.

[13] G. Brown, J. L. Wyatt, and P. Tino, "Managing diversity in regression ensembles," The Journal of Machine Learning Research, vol. 6, pp. $1621-1650,2005$

[14] J. V. Hulse, T. M. Khoshgoftaar, and A. Napolitano, "Experimental perspectives on learning from imbalanced data," in ICML '07: Proceedings of the 24th international conference on Machine learning, 2007, pp. 935-942.

[15] S. Wang and X. Yao, "Diversity analysis on imbalanced data sets by using ensemble models," in IEEE Symposium on Computational Intelligence and Data Mining 2009, 2009.

[16] S. Kotsiantis, D. Kanellopoulos, and P. Pintelas, "Handling imbalanced datasets: A review," GESTS International Transactions on Computer Science and Engineering, vol. 30, 2006.

[17] G. U. Yule, "On the association of attributes in statistics," Philosophical transactions of the Royal society of London, vol. A194, pp. 257$319,1900$.

[18] N. V. Chawla, K. W. Bowyer, L. O. Hall, and W. P. Kegelmeyer, "Smote: Synthetic minority over-sampling technique," Journal of Artificial Intelligence Research, pp. 341-378, 2002.

[19] A. Chandra and X. Yao, "Ensemble learning using multi-objective evolutionary algorithms," Journal of Mathematical Modelling and Algorithms, vol. 5, no. 4, pp. 417-445, 2006.

[20] L. I. Kuncheva and C. J. Whitaker, "Measures of diversity in classifier ensembles and their relationship with the ensemble accuracy," Machine Learning, vol. 51, pp. 181-207, 2003.

[21] G. Brown, J. Wyatt, R. Harris, and X. Yao, "Diversity creation methods: a survey and categorisation," Information Fusion, vol. 6, no. 1, pp. 5-20, 2004. 